\title{
PURIFICACIÓN Y ALGUNAS PROPIEDADES BIOQUÍMICAS Y MOLECULARES DE UNA NUEVA FOSFOLIPASA A2 NO MIOTÓXICA DEL VENENO DE LA SERPIENTE Bothrops atrox
}

Edwin Quispe ${ }^{1 *}$, Daniel Torrejón ${ }^{1}$, Lorgio Bautista ${ }^{1}$, Gustavo Sandoval $^{1}$, Edith Rodríguez ${ }^{1}$, Fanny Lazo ${ }^{1}$, Dan vivas-Ruiz ${ }^{1}$ y Armando Yarlequé ${ }^{1}$

\section{RESUMEN}

Las fosfolipasas $\mathrm{A}_{2}\left(\mathrm{PLA}_{2}\right)$ del veneno de las serpientes, son enzimas con una variedad de efectos biológicos, debido a sus diferentes isoformas y algunas pudiendo ser miotoxinas. El objetivo de la investigación fue purificar, caracterizar y evaluar la actividad miotóxica de una isoforma de PLA 2 ácida $\left(\right.$ BaPer-PLA $_{2 \mathrm{a}}$ ). Se purificó por DEAE Sephadex-A50, Sephadex-G75 y un sistema automatizado de presión media-NGC. La BaPer-PLA2a tuvo una actividad específica de 34,1 U/mg y un peso molecular de $\sim 14,5 \mathrm{kDa}$ por PAGE-SDS en condiciones no reductoras. Del veneno se obtuvo el ARN total, para la síntesis de ADNc y un amplificado de $\sim 480 \mathrm{pb}$. Se dedujo de la secuencia de ADNc una proteína madura de 124 aminoácidos con un punto isoeléctrico $(4,41)$, siendo una isoforma ácida, asimismo presentó una estructura primaria con regiones conservadas y los residuos His48, Asp49 y Tyr52 identificados en el centro catalítico. Adicionalmente, el modelo teórico estructural posee una identidad mayor al $70 \%$ con otras PLA2 ácidas. Finalmente, la BaPer-PLA2a no presenta actividad miotóxica, sin embargo, al combinarla con la isoforma de PLA2 básica incrementó la actividad miotoxina de esta última en 21,58\%.

Palabras clave: Bothrops atrox, veneno, purificación, fosfolipasa $\mathrm{A}_{2}$ ácida, miotoxicidad.

\section{PURIFICATION AND SOME BIOCHEMICAL AND MOLECULAR PROPERTIES OF AN NEW NOT MYOTOXIC PHOSPHOLIPASE A2 FROM Bothrops atrox SNAKE VENOM}

\begin{abstract}
Phospholipases $\mathrm{A}_{2}\left(\mathrm{PLA}_{2}\right)$ from snake venom are enzymes with highly variety of biological effects, due to their different isoforms and some may be myotoxins. This research aimed was to purify, characterize and evaluate the myotoxic activity of an isoform of acid PLA $\left(\right.$ BaPer-PLA $_{2 \mathrm{a}}$ ). For purification, in DEAE Sephadex-A50, Sephadex-G75 and an automated

\footnotetext{
${ }^{1}$ Laboratorio de Biología Molecular, Facultad de Ciencias Biológicas - Universidad Nacional Mayor de San Marcos. Lima-Perú.

*edwin.quispe@unmsm.edu.pe
} 
medium pressure system-NGC were used. BaPer-PLA2a had a specific activity of $34.1 \mathrm{U} /$ $\mathrm{mg}$ and a MW of $14.5 \mathrm{kDa}$ by SDS-PAGE, non-reducing conditions. From venom were obtained total RNA, to synthesis of cDNA and an amplified of $\sim 480 \mathrm{bp}$. A mature protein of 124 amino acids was deduced from the cDNA sequence with a pI (4.41), being an acidic isoform, likewise presented primary structure with conserved regions and residues His48, Asp49 and Tyr52 identified in the catalytic center. Additionally, the structural theoretical model has an identity greater than $70 \%$ with other acidic PLA2. Finally, BaPer-PLA2a did not have myotoxic activity, however, when combined with the basic PLA2 isoform increased the last 's myotoxic activity in $21.58 \%$.

Key words: Bothrops atrox, venom, purification, acidic phospholipase A2, myotoxicity.

\section{INTRODUCCIÓN}

Los venenos de las serpientes son una mezcla de distintas proteínas, las cuales le han permitido sobrevivir a lo largo del tiempo, ya que, mediante las mismas, han desarrollado un mecanismo de defensa y captura hacia su presa. En este veneno destacan las fosfolipasas $\mathrm{A}_{2}$ (E.C. 3.1.1.4), la cual hidroliza a los fosfolípidos en la posición n-2 del glicerol, liberando lisofosfolípidos y ácidos grasos ${ }^{3,8}$. Además, existen otras proteínas, tales como; metaloproteasas, serino proteasas, L-aminoácido oxidasas, hialuronidasas y proteína similar a trombina ${ }^{7}$, las cuales permiten que el veneno se difunda en el tejido, generando diversos efectos biológicos. La superfamilia de fosfolipasas $\mathrm{A}_{2}\left(\mathrm{PLA}_{2}\right)$ presenta una clasificación de acuerdo a su origen, secuencias aminoacídicas, estructura y función, pudiendo ser; secretoras, citosólicas, lisosomales, dependientes o independientes de calcio ${ }^{3}$.

En el Perú, la serpiente Bothrops atrox es la más peligrosa y causante de la mayoría de envenenamientos. No obstante, hay mucho interés en los componentes no sólo para generar un antiveneno sino porque presenta ciertas aplicaciones clínicas. Por ello, se realizó un estudio proteómico de tres serpientes peruanas del género Bothrops donde concluyen que la $\mathrm{PLA}_{2}$ es la segunda en abundancia en el veneno ${ }^{7}$. Además, se han estudiado a las PLA $\mathrm{P}_{2}$ en las serpientes, $L$. muta $^{6}$ y $B$. atrox ${ }^{5}$. Además, se caracterizó por primera vez a la miotoxina del veneno en $B$. brazili ${ }^{17}$. No obstante, aún no se ha investigado a las isoformas de PLA 2 ácidas en B. atrox ni su efecto en la miotoxicidad. Por ello, tal situación conduce a explorar en mayor profundidad este tipo de efecto biológico.

Las PLA 2 pertenece al grupo II, a su vez tienen dos subgrupos: Asp49(D49) y Lys49(K49). Dentro de cada subgrupo, hay a su vez isoformas pudiendo ser; Asp49(D49) ácidas 1,16,19,20, Asp(D49) básicas ${ }^{10,12}$ y Lys49 (K49) básicas ${ }^{10,12}$, las cuales tienen diversos efectos biológicos. $\mathrm{La}$ isoforma básica ha sido la más investigada, siendo altamente miotoxica ${ }^{5,10,12}$, en tanto que a la isoforma ácida también se le atribuye diversos efectos biológicos; miotóxica ${ }^{1,2,16,18}$, hemolítica indirecta ${ }^{16}$, inhibidora de agregación plaquetaria ${ }^{1,16,18}$ e hipotensiva $^{14,20}$. No obstante, de todos ellos, el efecto miotóxico es el más grave ya que puede dañar irreversiblemente el tejido muscular y dar lugar a amputaciones. Además, se ha reportado que la sinergia entre las miotoxinas D49 y K49, potencian el efecto miotóxico ${ }^{12}$. 
Por otro lado, la predicción estructural teórica terciaria de la isoforma de $\mathrm{PLA}_{2}$ ácida puede permitir entender la relación estructura-función durante el envenenamiento. Dentro de este panorama, la presente investigación estuvo enfocada en aislar a la primera isoforma de $\mathrm{PLA}_{2}$ ácida (BaPer-PLA $\mathrm{A}_{2 \mathrm{a}}$ ), conocer sus propiedades bioquímicas, moleculares, estructural y fundamentalmente su relación en el proceso miotóxico, el cual presenta gran importancia clínica, teniendo en cuenta que esta acción biológica es la menos sensible a la neutralización con el antiveneno comercial.

\section{PARTE EXPERIMENTAL}

\section{Muestras}

El veneno se obtuvo de la serpiente peruana Bothrops atrox "jergón" de la zona de Alto Marañón, departamento de Amazonas, mantenida en cautiverio en el serpentario "Oswaldo Meneses" - UNMSM. El veneno extraído fue liofilizado y conservado a $0^{\circ} \mathrm{C}$, hasta su empleo.

\section{Purificación de BaPer-PLA}

Se resuspendió $120 \mathrm{mg}$ de veneno liofilizado en acetato de amonio $50 \mathrm{mM}$ a pH 6,4, se centrifugó a $4000 \mathrm{rpm}$ por $20 \mathrm{~min}$ para eliminar restos insolubles. El sobrenadante fue aplicado a una columna cromatográfica de intercambio aniónico DEAE-Sephadex-A50 (16,5 $\mathrm{cm} \times 1,8 \mathrm{~cm}$ ) equilibrada con buffer de elución acetato de amonio $50 \mathrm{mM}$ a pH 6,4 a un flujo de $\sim 12 \mathrm{~mL} / \mathrm{h}$. Para eluir las proteínas ligadas a la resina se cambió el buffer isocrático a uno que contenía $\mathrm{NaCl} 0,3 \mathrm{M}$ y $0,6 \mathrm{M}$. Aquellas fracciones que mostraron actividad enzimática, fueron juntadas y concentradas para aplicarlas a una columna cromatográfica de exclusión molecular Sephadex-G75 (58 cm x 1,5 cm), equilibrada con el buffer acetato de amonio 50 $\mathrm{mM}$ a pH 6,4 a un flujo de $\sim 14 \mathrm{~mL} / \mathrm{h}$, colectando fracciones de $2 \mathrm{~mL}$ en un equipo colector (Pharmacia LKB SuperFrac). Finalmente, se empleó un sistema cromatográfico automatizado de presión media-NGC (Bio-Rad), utilizando una columna de intercambio anionico ENrichQ $(5 \times 50 \mathrm{~mm})$, equilibrada con el buffer de elución de acetato de amonio $50 \mathrm{mM}$ a pH 6,4 y se generó una gradiente de $\mathrm{NaCl}$ 0,1-1,0 M. Esta última corrida fue realizada a un flujo de 0,8 $\mathrm{mL} / \mathrm{min}$, colectando fracciones de $0,5 \mathrm{~mL}$.

\section{Cuantificación de proteínas}

Se realizó mediante dos formas; por absorbancia de luz UV a $280 \mathrm{~nm}$ con el espectrofotómetro Spectronic Genesys5 (Spectronic Ins) y por el método de Lowry ${ }^{11}$ empleando albúmina bovina como proteína estándar.

\section{Actividad enzimática}

Se determinó la actividad enzimática de la fosfolipasa A2 ácida empleando el método descrito por Vidal y Stoppani ${ }^{22}$. Se utilizó el sustrato en una relación (4:5) de yema de huevo y buffer Tris-HCl 10 mM (pH 7.4 y CaCl2 $10 \mathrm{mM}$ ), respectivamente. Se empleó 1,5 mL de sustrato con $100 \mu \mathrm{L}$ de veneno crudo o $50 \mu \mathrm{L}$ proteína purificada. La mezcla fue pre-incubada en un baño maría a $37^{\circ} \mathrm{C}$ por 15 min y se midió el retardo de coagulación de la yema de huevo a $100^{\circ} \mathrm{C}$. La actividad específica se expresó como el tiempo de retardo en la coagulación por minuto y miligramo de proteína $(\mathrm{U} / \mathrm{mg})$. 


\section{Electroforesis en gel de poliacrilamida con dodecil sulfato de sodio (PAGE-SDS)}

Se procedió de acuerdo a la metodología descrita por Laemmli ${ }^{9}$. La muestra fue incubada a $100^{\circ} \mathrm{C}$ y se preparó un gel acrilamida al $15 \%$. Se corrió en condiciones reductoras $(0,5 \%$ $\beta$-mercaptoetanol) y no reductoras a $100 \mathrm{~V}$ en un sistema electroforesis Mini-PROTEAN Tetra Cell, 4-Gel System (Bio-Rad). La tinción del gel fue con azul brillante de Coomassie R-250 por 15 min y la revelación de las bandas se obtuvo por decoloración con una mezcla de metanol, etanol, ácido acético glacial y agua en una relación de 2:1:5:6,5, respectivamente. Se empleó como patrón de bandas proteicas Page Ruler Prestained Protein Ladder (Thermo Scientific).

\section{Actividad miotóxica}

Se empleó ratones de la línea Balb/c (18-22g, $\mathrm{n}=3$ por grupo) del Instituto Nacional de Salud (INS), Lima-Perú. Los cuidados éticos de los animales fueron en base; International Guiding Principles for Biomedical Research Involving Animals (1985), by the Council for International Organizations of Medical Sciences (COIMS). En un primer grupo se inyectó en el músculo gastrocnemius, la enzima purificada BaPer-PLA ${ }_{2 \mathrm{a}}(30 \mu \mathrm{g} / 100 \mu \mathrm{L})$. En un segundo grupo fue inyectada la $\mathrm{PLA}_{2}$ básica miotóxica $(20 \mu \mathrm{g} / 100 \mu \mathrm{L})$. En un tercer grupo, la combinación entre la BaPer-PLA ${ }_{2 \mathrm{a}}$ y la $\mathrm{PLA}_{2}$ básica miotóxica $(5 \mu \mathrm{g}+20 \mu \mathrm{g} / 100 \mu \mathrm{L})$ y como control negativo se empleó $100 \mu \mathrm{L}$ de solución salina. Posterior a las tres horas de la inoculación, se colectó sangre de la vena facial que fue recibida en tubos de 1,5 mL, conteniendo $40 \mu \mathrm{L}$ de citrato de sodio al 3,8 \%. La sangre fue centrifugada a $2000 \mathrm{rpm}$ por 10 min para la liberación del plasma con creatina quinasa (CK). Se utilizó el kit CK-NAC FS* (DiaSys, GmbH-Germany), donde se empleó $40 \mu \mathrm{L}$ de plasma con $1 \mathrm{~mL}$ del kit. La lectura de absorbancia fue a $340 \mathrm{~nm}$, realizada en un espectrofotómetro Genesys5 (Spectronic Ins.) y la actividad creatina quinasa (CK) fue expresada en (U/L).

\section{Diseño de cebadores}

Los cebadores se diseñaron a partir de un alineamiento múltiple de las secuencias de PLA2 ácidas depositadas en el Gen Bank correspondiente a las especies: B. moojeni (HQ327311.1), B. neuwiedi pauloensis (GQ850458.1), B. asper (MF319968.1), B. jararacussu (AY145836.1), B. diporus (JQ661499.1; JQ661500.1) y de B. erythromelas (DQ359953.1). Los cebadores fueron diseñados manualmente tomando en cuenta las regiones no traducidas (UTRs) ubicados en los extremos del gen (F1BAPLA2 -R1BAPLA2) y sintetizados por el fabricante (Invitrogen).

\section{Aislamiento de ARN total}

Se empleó muestras de veneno liofilizado de B. atrox y siguiendo el protocolo del fabricante TRIzol $^{\mathrm{TM}}$ Reagent (Invitrogen): Se agregó $1 \mathrm{ml}$ de TRIzol a $40 \mathrm{mg}$ de muestra y se homogenizó por $5 \mathrm{~min}$. Después, se agregó 0,2 $\mathrm{mL}$ de cloroformo, se incubó a temperatura ambiente por 3 min y se centrifugó a $12.000 x g$ por 15 min. Luego, se transfirió la fase acuosa a otro tubo, se agregó $0,5 \mathrm{~mL}$ de isopropanol, se incubó por 10 min a temperatura ambiente y se centrifugó a 12.000xg por $10 \mathrm{~min}$. Se descartó el sobrenadante y el pellet fue lavado con un $1 \mathrm{ml}$ de etanol al $75 \%$. De inmediato, se descartó el etanol y se secó el pellet por inversión. Finalmente, el ARN total fue resuspendido en $50 \mu \mathrm{L}$ de agua ultra pura (Invitrogen) libre de 
ARNasa y se dejó incubar en un AccuBlock Digital Dry Bath (Labnet) a $58^{\circ} \mathrm{C}$ por $15 \mathrm{~min}$. La cuantificación se realizó en un equipo Nanodrop Lite Spectrofotometre (Thermo Scientific), para calcular la concentración y calidad del ARN total.

\section{Síntesis de ADNc}

En la síntesis se siguió el protocolo del fabricante SuperScript ${ }^{\mathrm{TM}}$ III Reverse Transcriptase (Invitrogen). Se partió de 6,66 ng de ARN total, luego se agregó 400 ng de oligo (dT) ${ }^{18-20}, 9,2$ $\mu \mathrm{L}$ de agua miliQ, $1 \mu \mathrm{L}$ de mix dNTPs $10 \mathrm{mM}$ c/u. La mezcla se incubó a $65^{\circ} \mathrm{C}$ por 5 min y un 1 min en frío, se centrifugó brevemente y se agregó $4 \mu \mathrm{L}$ de buffer First-Strand ( $5 \mathrm{X}), 1 \mu \mathrm{L}$ de DTT 0.1 M, $1 \mu \mathrm{L}$ de SuperScript ${ }^{\mathrm{TM}}$ III RT (200 units/ $\mu \mathrm{L}$ ). Después, la mezcla se homogenizó y se incubó a $50^{\circ} \mathrm{C}$ por 45 min. Posteriormente, para la inactivación de la reacción se incubó a $70^{\circ} \mathrm{C}$ por $15 \mathrm{~min}$. La cuantificación se realizó en un equipo Nanodrop Lite Spectrofotometre (Thermo Scientific), para calcular la concentración y calidad de ADNc.

\section{Amplificación de la región codificante de la PLA2 ácida por PCR}

La amplificación del gen de la PLA2 por reacción en cadena de la polimerasa por sus siglas en inglés (PCR) fue realizada en un termociclador Mastercycler (Eppendorf). La reacción tuvo $100 \mathrm{ng}$ de $\mathrm{ADNc}$, agua mili Q, dNTPs $0.2 \mathrm{mM}, \mathrm{MgCl}_{2} 2 \mathrm{mM}$, tampón buffer con $\mathrm{KCl}$ $1 \mathrm{X}, 0.4 \mu \mathrm{M}$ de cada cebador forward (5'-ATGAGGACTCTCTGGATAGTGA-3'), reverse (5'-TTAGCATGGCTCTGCCTTCT-3') y 0,3 $\mu \mathrm{L}$ de Taq Polimerase Recombinant (5 U/ $\mu \mathrm{L})$. Las condiciones del amplificado fueron: desnaturalización inicial a $95^{\circ} \mathrm{C}$ por 3 min, desnaturalización a $95^{\circ} \mathrm{C}$ por $40 \mathrm{seg}$, alineamiento a $54^{\circ} \mathrm{C}$ por $45 \mathrm{seg}$ y extensión $72^{\circ} \mathrm{C}$ por 1 min, por 33 ciclos y extensión final a $72^{\circ} \mathrm{C}$ por $6 \mathrm{~min}$. El producto de PCR fue corrido en un gel de agarosa $(1,2 \%)$ con buffer TBE $1 \mathrm{X}$ en un sistema de electroforesis (Cleaver Scientific Ltd), y el amplicón fue estimado con el Ladder GenRuler 100 pb DNA (Thermo scientific). Luego, el gel fue teñido con GelRed $(0,01 \%)$ y observado en un UV Transilluminator/ microDoc (Cleaver Scientific Ltd). Los productos fueron secuenciados por EZ-Seq, realizado en la empresa Macrogen, Inc (Corea del Sur).

\section{Análisis bioinformático}

Para la identificación de la secuencia obtenida se usó las bases de datos disponibles en National Center for Biotechnology Information (https://www.ncbi.nlm.nih.gov/), UniProt (https://www.uniprot.org/) y para la identificación de estructuras homólogas se basó Protein Date Bank (www.rcsb.org). Se utilizó el programa (ClustalW) para alineamiento y generar la secuencia consenso de nucleótidos, de la cual se basó para obtener la estructura primaria de aminoácidos con Translate tool (https://web.expasy.org/translate/), Asimismo, se determinó el punto isoeléctrico con el programa ProtParam (https://web.expasy.org/protparam/). Por otro lado, también se emplearon los siguientes programas; Robetta (new.robetta.org) para el modelo comparativo, Modeller (https://salilab.org/modeller/) para la homología estructura y $\mathrm{PyMol}^{22}$ para el modelamiento tridimensional de BaPer-PLA ${ }_{2 \mathrm{a}}$. 


\section{RESULTADOS Y DISCUSIÓN}

\section{Purificación y determinación del peso molecular de BaPer-PLA $A_{2 a}$.}

La presente enzima purificada denominada BaPer-PLA ${ }_{2 a}$, es la primera isoforma ácida en ser aislada del veneno de la especie $B$. atrox de Perú. En la primera etapa de purificación por intercambio aniónico, las proteínas fueron separadas por su carga. La enzima de interés eluyó después de adicionar $\mathrm{NaCl}$ 0,3 M, estando registrada en la caída del cuarto pico (figura 1A). El pool conteniéndola fue sometido a una segunda etapa de purificación por exclusión molecular, la cual permitió separar a la enzima por diferencia de pesos; la BaPer-PLA2a fue registrada en la caída del tercer pico (figura 1B). Posteriormente, se empleó el sistema cromatográfico automatizado de presión media -(NGC), empleando una gradiente de $\mathrm{NaCl}$ 0,1-1,0 M para separar a los contaminantes residuales, la cual permitió obtener a BaPer-PLA2a en el tercer pico (figura 1C), verificando que se encontró la enzima en el estado homogéneo, como lo indica en análisis por PADE-SDS (figura 1D). En la tabla 1 se muestra los procesos de purificacion, en la primera etapa de purificacion las unidades totales de actividad (UTA) se incrementaron en $37 \%$ lo que indica la probable liberación de un inbihidor endógeno de dicha enzima. Finalmente, se logró obtener la enzima, a una purificación de 14,82 veces y un rendimiento de $3,39 \%$.
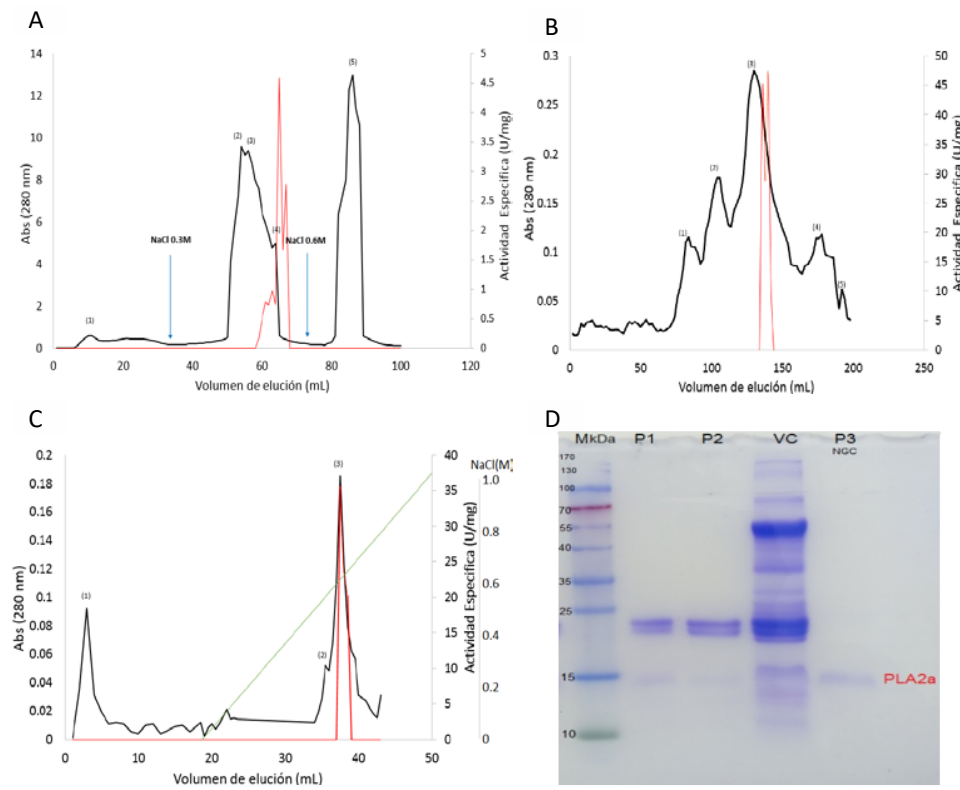

Figura 1. Cromatogramas de purificación. En (A) se muestra la primera etapa, la línea roja muestra la actividad enzimática específica. En (B) la segunda etapa, la actividad específica alcanza un valor de 45 (U/mg). En (C) la tercera etapa de purificación, en línea verde indica la gradiente de $\mathrm{NaCl}$ y la enzima en estudio se registra en el tercer pico, mientras que la línea roja indica la actividad específica de BaPer-PLA2a con un valor de 34,1 U/mg. En (D) Se muestra un PAGE-SDS, en condiciones no reductoras. P1 y P2 corresponden a muestras del segundo paso de purificación, en tanto que P3, después del paso por el sistema cromatográfico automatizado. Se llegó a obtener

la proteína en su estado homogéneo, con peso de $\sim 14,5 \mathrm{kDa}$. VC indica veneno total y (M) el marcador de peso molecular. 
El uso combinado de sistemas cromatográficos ha sido la estrategia más eficaz para la purificación de las $\mathrm{PLA}_{2}$ en especies tales como: la $\mathrm{PLA}_{2}$ ácida en $B$. pauloensis ${ }^{1}$ la $\mathrm{PLA}_{2}$ básica miótoxica en $B$. atrox ${ }^{5}$ y PLA $_{2}$ ácida en $B$. moojeni ${ }^{19}$.

El peso molecular de la enzima analizada por PAGE-SDS fue de $\sim 14.5 \mathrm{KDa}$ en condiciones no reductoras (figura 1D). Este resultado es compatible con otras PLA ácidas como; en B. pauloensis $(15 \mathrm{kDa})^{1}$, en $B$. moojeni $(15,8 \mathrm{kDa})^{18}$ y en $B$. asper $(\sim 14,5 \mathrm{kDa})^{16}$. Por lo tanto, la enzima se trata de proteína de bajo peso molecular. Además, el tratamiento con $\beta$-mercaptoetanol sugiere que es monomérica, este resultado es compatible con otros trabajos ${ }^{1,14}$.

Tabla 1. Purificación de la enzima BaPer-PLA2a del veneno de Bothrops atrox.

\begin{tabular}{lcccccc}
\hline \multirow{2}{*}{ Etapas } & \multicolumn{2}{c}{ Proteína Total } & A.E. & U.T.A. & $\begin{array}{c}\text { Rendimiento } \\
\%\end{array}$ & Purificación \\
\cline { 2 - 4 } & $\mathrm{mg}$ & $\%$ & $(\mathrm{U} / \mathrm{mg})$ & & 100 & 1 \\
\hline Inicial: Veneno crudo & 120 & 100 & 2,3 & 276 & 137 & 3,1 \\
\hline $1^{\circ}:$ DEAE-Sephadex A50 & 52,98 & 44,15 & 7,14 & 378,27 & 21,96 & 7,1 \\
\hline $2^{\circ}:$ Sephadex G75 & 3,709 & 3,09 & 16,34 & 60,6 & 3,39 & 14,82 \\
\hline $3^{\circ}:$ ENrichQ & 0,275 & 0,229 & 34,1 & 9,377 & \\
\hline
\end{tabular}

A.E.: Actividad específica (U/mg de proteína), determinado por el método yema de huevo ${ }^{22}$ U.T.A: Unidades totales de actividad.

\section{Actividad enzimática de BaPer-PLA}

La medición de la actividad enzimática se realizó usando la prolongación del tiempo de coagulación de emulsión de yema de huevo de Vidal y Stoppani ${ }^{21}$, en este caso se obtuvo una actividad específica de 34,1 U/mg lo que equivale a una purificación de 14,82 veces comparado con el veneno total (tabla 1). Esto se debió a la hidrólisis de los fosfolípidos a nivel del enlace éster $\mathrm{n}-2$, liberando lisofosfolípidos y ácidos grasos ${ }^{3,8}$.

Si bien existen métodos más sensibles para medir la actividad de PLA ${ }_{2}$, el método de yema de huevo permitió hacer el seguimiento rápido de la enzima en cada etapa de purificación. Adicionalmente, se realizó la medición de actividad con el sustrato fosfatidilcolina de la enzima purificada, obteniendo una actividad de 179,6 U/mg.

Mucho se ha discutido acerca de la validez del método enzimático de prolongación del tiempo de coagulación de la yema de huevo ya que se trata de un sustrato heterogéneo. Sin embargo, el método es absolutamente idóneo para fines prácticos teniendo resultados reproducibles. Por otro lado, el método por espectrometría usado con el sustrato fosfatidilcolina tiene una alta sensibilidad, pero el procedimiento es laborioso en términos de aplicación, aunque se requiere pocas cantidades de enzima para el ensayo ${ }^{4,15}$. 


\section{Actividad miotóxica de BaPer-PLA ${ }_{2 a}$.}

La enzima por sí sola no mostró actividad miotóxica al obtener valores iguales o menores al blanco durante la liberación de creatina qQuinasa (CK). Sin embargo, cuando es BaPer-PLA es combinada con la isoforma de $\mathrm{PLA}_{2}$ básica miotóxica; el valor inicial de la isoforma de $\mathrm{PLA}_{2}$ básica de $740 \mathrm{U} / \mathrm{L} \pm 0,07$, aumenta a $915 \mathrm{U} / \mathrm{L} \pm 0,065$, lo que equivale a un incremento del $21,58 \%$ de la actividad miotóxica (figura 2). La carencia de actividad miotóxica de BaPer-PLA $_{2 \mathrm{a}}$ se ha reportado en otras isoformas ácidas del grupo II19, no obstante, también se ha reportado que la isoforma de $\mathrm{PLA}_{2}$ ácida es capaz de producir una liberación alta o moderada de creatina quinasa $(\mathrm{CK})^{1,16,18}$.

Un hecho relevante en esta investigación fue el hallazgo del incremento de la actividad miotóxica cuando se combinan las isoformas de $\mathrm{PLA}_{2}$ ácida y básica, lo que sugiere que durante el envenenamiento podría ocurrir un incremento del daño al tejido muscular por acción sinérgica de ambas isoformas. Asimismo, esto abre un camino para intentar no sólo bloquear a la $\mathrm{PLA}_{2}$ básica miotóxica, sino también a la enzima BaPer-PLA $2 \mathrm{a}$. Este resultado es compatible con lo reportado en la combinación de D49 y K49 ${ }^{12}$.

PLA2 b. M. y BaPer-PLA2a

PLA2 b. M.

BaPer-PLA2a

Blanco

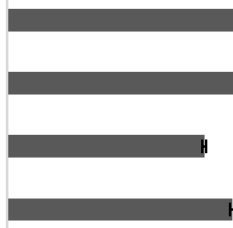

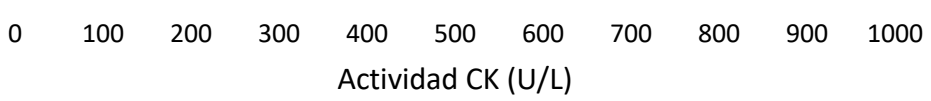

Figura 2. Actividad creatina quinasa (CK). La PLA 2 básica miotóxica $(20 \mu \mathrm{g} / 100 \mu \mathrm{L})$, la BaPerPLA $_{2 \mathrm{a}}(30 \mu \mathrm{g} / 100 \mu \mathrm{L})$, la combinación de PLA básica miotóxica con BaPer-PLA $_{2 \mathrm{a}}(20 \mu \mathrm{g}+5 \mu \mathrm{g}$ $/ 100 \mu \mathrm{L})$ y el blanco $100 \mu \mathrm{L}$ de solución salina.

\section{Aislamiento de ARN total, síntesis de ADNc y amplificación por PCR}

De $40 \mathrm{mg}$ de veneno liofilizado, se aisló $77 \mathrm{ng} / \mu \mathrm{L}$ de ARN total, por otro lado, a partir de $65 \mathrm{mg}$ tejido glandular, se obtuvo $1282,4 \mathrm{ng} / \mu \mathrm{L}$ de ARN total. Las glándulas del veneno representan una fuente primaria para obtención de altas concentraciones de ARN total ${ }^{1,16}$. No obstante, esto implica el sacrificio del espécimen que para efectos de la presente investigación no es óptimo y atenta contra los principios de conservación de la especie.

Para la síntesis de ADNc se obtuvo $541 \mathrm{ng} / \mu \mathrm{L}$, a partir de veneno liofilizado y $762,5 \mathrm{ng} / \mu \mathrm{L}$ de tejido glandular. Por otra parte, en la amplificación del gen de BaPer-PLA $A_{2 a}$ se obtuvo un producto de $\sim 480 \mathrm{pb}$ (figura 3), tamaño que es comparable a otros amplificados de $448 \mathrm{pb}$ en L. muta $^{6}, 417 \mathrm{pb}$ de B. pauloensis ${ }^{1}$ y de $414 \mathrm{pb}$ en B. moojeni ${ }^{19}$. 


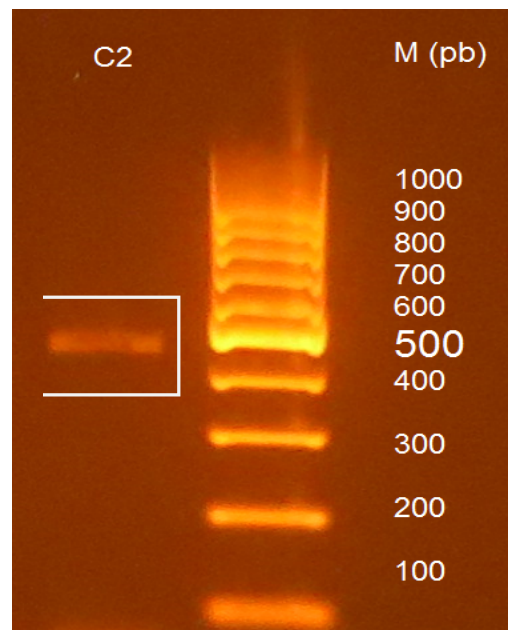

Figura 3. Electroforesis en agarosa $1 \%$. En $\mathrm{C} 2$ se muestra en el cuadro en blando el amplificado con un peso de $\sim 480 \mathrm{pb}$. Siendo M el marcador en pares de bases (100-1000 pb).

\section{Análisis bioinformático de la BaPer-PLA 2 .}

La estructura primaria de la enzima presenta 124 aminoácidos deducidos a partir de la secuencia de nucleótidos obtenida posterior a la amplificación. Los análisis por Blastp (NCBI)

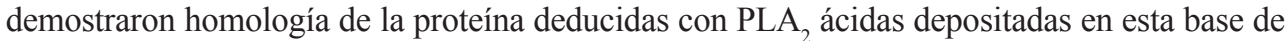
datos. Posteriormente, el alineamiento múltiple con las PLA ácidas de 11 especies del genero Bothrops mostró que la BaPer-PLA ${ }_{2 \mathrm{a}}$ presenta una identidad entre $70-84 \%$ con sus semejantes (figura 4). Asimismo, existe $83 \%$ de identidad con la PLA 2 ácida de B. asper de Colombia, esto puede ser debido a la cercanía taxonómica entre ambas especies amazónicas. La BaPer$\mathrm{PLA}_{2 \mathrm{a}}$ presentan 14 aminoácidos cisteínas altamente conservados, las cuales formarían los siete enlaces disulfuro, mientras que los cuatro aminoácidos en las posiciones (Tyr28, Gly30, Gly32 y Asp49) formarían el loop de unión al calcio y un centro catalítico constituido por los residuos (His48, Asp49, Tyr52 y Asp99) (figura 4). Además, se dedujo su punto isoeléctrico (pI) teórico de 4,41 .

Esto permitió concluir que se trata de una isoforma de $\mathrm{PLA}_{2}$ ácida.

Estos resultados son similares a los reportados en B. insulares $^{2}$, B. asper $^{17}$, B. pauloensis $^{1}$ y B. moojeni ${ }^{18,19}$. Además, son regiones altamente conservadas en las fosfolipasas $\mathrm{A}_{2}$ ácidas pertenecientes al grupo II de las serpientes de la familia Viperidae.

EL análisis bioinformático coloca en cercanía de congruencia de identidad a la secuencia proteica deducida con la proteína purificada en el presente trabajo, esto porque los cebadores fueron diseñados partiendo de la región conservadas de los UTR 5 `y $3^{`}$ de las secuencias de las $\mathrm{PLA}_{2}$ ácidas con mayor cercanía taxonómica a la especie estudiada. 


\begin{tabular}{|c|c|c|c|c|c|}
\hline BaPer-PLA2a & \multicolumn{4}{|c|}{1 SLVAFEMMIMDVMRKNVVLFYLSYGCYCGWGGLGRPQDATDRCCFVHDCCYGKVTDCDPKTDIYTYTYDN } & 70 \\
\hline Q8AXY1 & \multicolumn{5}{|c|}{1 SLWQFGKMINYVMGESGVLQYLSYGCYCGLGGQGQPTDATDRCCFVHDCCYGKVTGCDPKID--SYTYSK 68} \\
\hline Q8QG87 & \multicolumn{4}{|c|}{1 NLWQFGKMMNYVMGQSVVYKYFYYGCYCGWGGIGQPRDATDRCCFVHDCCYGKVTGCDPKTD--SYTYSK } & 68 \\
\hline Q2HZ28 & \multicolumn{5}{|c|}{1 SLVQFETLIMKIAGRSGVWYYGSYGCYCGSGGQGRPQDASDRCCFVHDCCYGKVTDCDPKAD--VYTYSE 68} \\
\hline & \multicolumn{5}{|c|}{1 NLWQFEMLIMKIAKTSGFLFYSSYGCYCGWGGHGRPQDATDRCCFVHDCCYGKVTGCNPKTD--SYTYSE 68} \\
\hline & \multicolumn{5}{|c|}{1 NLWQFGKLIMKIAGESGVFKYSYGCYCGLGGQGQPTDATDRCCFVHDCCYGKVTGCDPKID--SYTYSK 68} \\
\hline & \multicolumn{5}{|c|}{1 NLWQFGQMMSDVMRKNVVEKYLSYGCYCGWGGIGQPKDATDRCCFVHDCCYGKVTGCDPKMDIYTYTYSK 70} \\
\hline & \multicolumn{5}{|c|}{1 DLWQFGQMMNDVMREYVVFNYLYYGCYCGWGGIGKPRDATDRCCFVHDCCYGKVTGCNPKTDSYTYTYSE 70} \\
\hline 29 & \multicolumn{5}{|c|}{1 SLVQFETLIMKIAKRSGVWFYGSYGCFCGSGGQGRPQDASDRCCFVHDCCYGKVTDCDPKTD--IYTYSE } \\
\hline 581 & \multicolumn{5}{|c|}{1 NLVQFGEMMSDVMRKNVVEKYLSYGCYCGWGGLGRPQDATDRCCFVHDCCYGKVTGCDPKTDIDTYTYSE 70} \\
\hline 84 & \multicolumn{5}{|c|}{1 SLVQFDTLIMKIAGRSGIIFYSSYGCYCGLGGQGRPQDASDRCCFVHDCCYGKVTGCNPKAD--VYTYSE } \\
\hline 08 & \multicolumn{5}{|c|}{1 NLVQFETLIMKIAGRSGVWYYGSYGCYCGSGGQGRPQDASDRCCEVHDCCYGKVTGCNPKAD--TYTYSE 68} \\
\hline us & \multicolumn{5}{|c|}{ 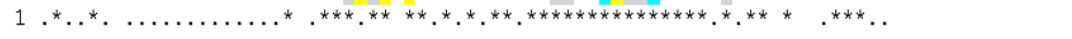 } \\
\hline & \multicolumn{5}{|c|}{ Especie } \\
\hline $\mathrm{r}-\mathrm{PLA} 2 \mathrm{a}$ & \multicolumn{5}{|c|}{71 ENGDVVCGGDDPCQNHICDCDRVAATCFRDDKDTYDSKYWFYGAKNCQEESEPC 124} \\
\hline & 69 & KNGDVVCGGDDPCKKQICECDRVATTCFRDNKDTYDIKYWFYGAKNCQEKSEPC 122 & $75.00 \%$ & \multicolumn{2}{|c|}{ B. jararacussu } \\
\hline & 69 & ENGDVVCGGDDPCKKQICECDRVAATCFRDNKDTYDMKYWLYGAKNCQEESEPC 122 & $75.00 \%$ & \multicolumn{2}{|c|}{ B. insularis } \\
\hline & 69 & ENGVVVCGGDDPCKKQICECDRVAATCFRDNKDTYDNKYWFFPAKNCQEESEPC 122 & $75.00 \%$ & \multicolumn{2}{|c|}{ B. erythromelas } \\
\hline & 69 & ENGDVVCGGDDPCKKQICECDRVAATCFRDNKDTYDNKYWFYPAKNCQEESEPC 122 & $77.42 \%$ & \multicolumn{2}{|c|}{ B. moojeni } \\
\hline & 69 & ENGDVVCGGDDPCKKQICECDRVAATCFRDNKDTYDIKYWFYGAKNCQEESEPC 122 & $74.19 \%$ & \multicolumn{2}{|c|}{ B. pirajai } \\
\hline & 71 & ENGDVVCGGDDPCKKQICECDRVAAICFRDNKDTYDSKYWFYGAKNCQEDSEPC 124 & $81.45 \%$ & \multicolumn{2}{|c|}{ B. asper } \\
\hline & 71 & ENGDVVCGGDDLCKKQICECDRVAATCFRDNKDTYDTKYWLYGAKNCQEESEPC 124 & $78.05 \%$ & \multicolumn{2}{|c|}{ B. jararaca } \\
\hline & 69 & ENGVVVCGGDDPCKKQICECDRVAAVCFRDNKDTYDNKYWFFPANNCQEESEPC 122 & $75.00 \%$ & \multicolumn{2}{|c|}{ B. pictus } \\
\hline 581 & 71 & ENGDLVCGGDDPCKKQICECDRVAAICFRDNKDTYDIKYVFYGAKNCQEESEPC 124 & $83.06 \%$ & \multicolumn{2}{|c|}{ B. asper } \\
\hline & 69 & DNGDIVCGGDDPCKKQICECDRVAATCFRDNKDTYDNKYWLFPAKNCQEESEPC 122 & $71.77 \%$ & \multicolumn{2}{|c|}{ B. neuwiedi } \\
\hline & 69 & ENGVVVCGGDDPCKKQICECDRVAATCFRDNKDTYDNKYWFFPAKNCQEESEPC 122 & $73.39 \%$ & \multirow{2}{*}{\multicolumn{2}{|c|}{ B. diporus }} \\
\hline & & $* * * \ldots \ldots *$ & & & \\
\hline
\end{tabular}

Figura 4. El alineamiento múltiple de la estructura primaria de BaPer-PLA 2 a con otras fosfolipasas A2 ácidas de varias serpientes del género Bothrops. Las accesiones de las proteínas se muestran en la primera columna. Se muestra el número de aminoácidos, porcentaje de identidad y la especie de origen. El aminoácido de cisteína se muestra en color gris. El loop para la unión al calcio se muestra en color amarillo, en tanto que los aminoácidos del centro catalítico se muestran en color rojo-cian.

\section{Modelamiento estructural de la BaPer-PLA}

El modelamiento estructural se realizó en base a homología con las estructuras cristalizadas de la BthA-I-PLA2 de B. Jararacussu ${ }^{13,14}$ (PDB: 1ZLB, 1 U73 y 1UMV), los cuales tiene una identidad del 70-75 \% con la BaPer-PLA2a empleando el programa Modeller y generado en el programa PyMol ${ }^{22}$. La estructura construida (figura 5) presenta tres $\alpha$-hélice, un lazo- $\beta$, un loop para la unión al calcio y un centro catalítico (His48, Asp49, Tyr52 y Asp99) ${ }^{20}$. Además, este modelo también fue reproducido por otras investigaciones como la de BmooPLA2 en B. moojeni $^{21}$. 


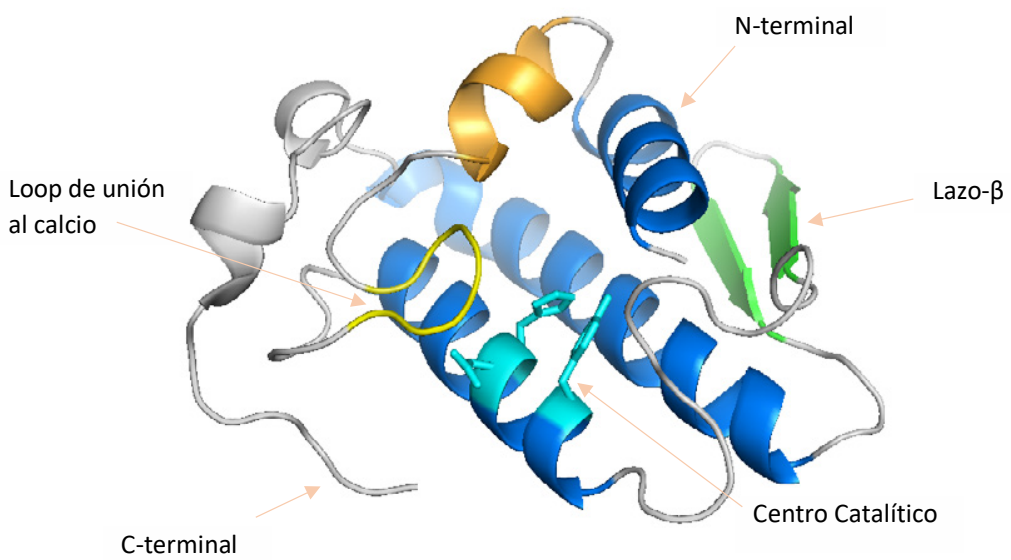

Figura 5. Se muestra el modelo teórico estructural de la BaPer-PLA $A_{2 a}$ desarrollado por el programa $\mathrm{PyMol}^{23}$. En amarillo se indica el loop para la unión al calcio. En cian el centro catalítico. En verde se indica el lazo- $\beta$ y en color azul las tres $\alpha$-hélice.

\section{CONCLUSIONES}

Esta investigación permitió purificar la primera isoforma ácida de PLA 2 (BaPer-PLA2a) a partir del veneno de la serpiente $B$. atrox que habita en la selva peruana. Posee actividad enzimática sobre lipoproteínas de yema de huevo y fosfatidilcolina, pero carece de actividad miotóxica. Su peso molecular fue de $\sim 14,5 \mathrm{kDa}$ en condiciones no reductoras y su $\mathrm{pI}(4,41)$, calculado in silico. La enzima incrementa la actividad miotóxica en 21,58 \% de su homóloga $\mathrm{PLA}_{2}$ básica, la cual es una miotoxina. Asimismo, se obtuvo una secuencia proteica deducida cercana a las características de la proteína purificada la cual pertenece al subgrupo (D49), presenta una estructura primaria de 124 aminoácidos, un centro catalítico formado por los residuos (His48, Asp49, Tyr52 y Asp99) y un loop para la unión al calcio. Finalmente, la enzima mostró una homología estructural teórica del 74,5 \% con 1ZLB.

Por otro lado, la investigación demostró que la isoforma ácida potencia el efecto de miotoxicidad elevando los niveles de daño al tejido muscular, lo cual sugiere que es importante considerar a la BaPer-PLA2a durante el desarrollo de algún antiveneno, mientras que el análisis de la estructura primaria permitió, también, identificar regiones variables, las cuales podrían ser atribuidas al aumento de la miotoxicidad cuando se combinan con la isoforma básica. Finalmente, el modelo teórico estructural permitió una comprensión parcial de la enzima y dejando abierta la posibilidad de estudios posteriores. 


\section{AGRADECIMIENTOS}

Los autores agradecen a FONDECYT- CONCYTEC por el financiamiento otorgado a través del contrato $\mathrm{N}^{\circ} 168-2017-F O N D E C Y T$ y a la UNMSM por las facilidades del laboratorio brindadas. Este trabajo es parte de la tesis de Magister en Biología Molecular del autor principal (Edwin Quispe Cerón). Se agradece al Bach. Alex Proleon por proporcionar gentilmente muestras de la isoforma de $\mathrm{PLA}_{2}$ básica miotóxica.

\section{REFERENCIAS BIBLIOGRÁFICAS}

1. Ferreira FB, Gomes MS, de Souza DL, Gimenes SN, Castanheira LE, Borges MH, et al. Molecular cloning and pharmacological properties of an acidic PLA2 from Bothrops pauloensis snake venom. Toxins (Basel). 2013; 5(12):2403-2419.

2. Cogo JC, Lilla S, Souza GH, Hyslop S, de Nucci G. Purification, sequencing and structural analysis of two acidic phospholipases A2 from the venom of Bothrops insularis (jararaca ilhoa). Biochimie. 2006; 88(12):1947-1959.

3. Dennis EA, Cao J, Hsu Y-H, Magrioti V, Kokotos G. Phospholipase A2 Enzymes: Physical Structure, Biological Function, Disease Implication, Chemical Inhibition, and Therapeutic Intervention. Chem Rev. 2011; 111(10):6130-6185.

4. Lazo F, Rodríguez E, Yarlequé A. Evaluación comparativa de dos métodos para determinar la actividad de fosfolipasa A en venenos de serpientes. Rev Perú Biol. 1998; 5(2):98-102

5. Huatuco S, Escobar E, Yarlequé A. Aislamiento y caracterización parcial de una miotoxina del veneno de la serpiente Bothrops atrox (Ophidia: Viperidae). Rev Perú Biol. 2004; 11(1): 79-86

6. Jiménez K, Zavaleta A, Izaguirre V, Yarlequé A, Inga R. Clonaje y caracterización molecular in silico de un transcrito de fosfolipasa A2 aislado del veneno de la serpiente peruana Lachesis muta. Rev Perú Med Exp Salud Pública. 2010; 27(4): 532-539.

7. Kohlhoff M, Borges MH, Yarlequé A, Cabezas C, Richardson M, Sánchez EF. Exploring the proteomes of the venoms of the Peruvian pit vipers Bothrops atrox, B. barnetti and B. pictus. J Proteomics. 2012; 75: 2181-2195

8. Kini MR. Excitement ahead: structure, function and mechanism of snake venom phospholipase $\mathrm{A}_{2}$ enzymes. Toxicon. 2003; 42: 827-840.

9. Laemmli UK. Cleavage of Structural Protein during the Assembly of the Head of Bacteriophage T4. Nature. 1970. 227: 680-685.

10. Lomonte B, Gutiérrez JM. Phospholipases A From Viperidade Snake Venoms: How do They Induce Skeletal Muscle Damage? Acta Chim Slov. 2011;58: 647-659.

11. Lowry OH, Rosebrough NJ, Farr AL, Randall RJ. Protein Measurement with the Folin Phenol Reagent. J Biol Chem. 1951; 193: 265-275.

12. Mora-Obando D, Fernández J, Montecucco C, Gutiérrez JM, Lomonte B. Synergism between basic Asp49 and Lys49 phospholipase A2 myotoxins of viperid snake venom in vitro and in vivo. PLoS One. 2014 Oct 7;9(10):e109846. doi: 10.1371/journal. pone.0109846. 
13. Murakami MT, Gabdoulkhakov A, Genov N, Cintra ACO, Betzel C, Arni RR. Insights into metal ion binding in phospholipase $\mathrm{A}_{2}$ : Ultra high-Resolution crystal Structures of an acidic phospholipase $\mathrm{A}_{2}$ in the $\mathrm{Ca}^{2+}$ free and bound states. Biochimie. 2006; 88: 543 549.

14. Magro AJ, Murakami MT, Marcussi S, Soares AM, Arni RK, Fontes MR. Crystal structure of an acidic platelet aggregation inhibitor and hypotensive phospholipase A2 in the monomeric and dimeric states: insights into its oligomeric state. Biochem Biophys Res Commun. 2004; 323(1):24-31.

15. Oliveira MR, Palma MS. Polybiotoxins: a group of phospholipases A2 from the venom of the neotropical social wasp paulistinha (Polybia Paulista). Toxicon. 1998; 36(1): 189199.

16. Posada Arias S, Rey-Suárez P, Pereáñez J A, Acosta C, Rojas M, Delazari Dos Santos $\mathrm{L}$, et al. Isolation and Functional Characterization of an Acidic Myotoxic Phospholipase $\mathrm{A}_{2}$ from Colombian Bothrops asper Venom. Toxins (Basel). 2017;9(11). pii: E342. doi: 10.3390/toxins9110342.

17. Pantigoso C, Escobar E, Yarlequé A. Aislamiento y caracterización de una miotoxina del veneno de la serpiente Bothrops brazili Hoge, 1953 (Ophidia: Viperidae). Rev Perú Biol. 2001; 8(2): 106-148

18. Santos-Filho NA, Silveira LB, Oliveira CZ, Bernardes CP, Menaldo DL, Fuly AL, et al. A new acidic myotoxic, anti-platelet and prostaglandin $\mathrm{I}_{2}$ inductor phospholipase $\mathrm{A}_{2}$ isolated from Bothrops moojeni snake venom. Toxicon. 2008; 52: 908-917.

19. Silveira LB, Marchi-Salvador DP, Santos-Filho NA, Silva FP Jr, Marcussi S, Fuly AL, et al. Isolation and expression of a hypotensive and anti-platelet acidic phospholipase A2 from Bothrops moojeni snake venom. J Pharm Biomed Anal. 2013; 73:35-43.

20. Scott DL, White SP, Otwinowski Z, Yuan W, Gelb MH, Sigler PB. Interfacial catalysis: the mechanism of phospholipase A2. Science. 1990; 250(4987):1541-1546.

21. Vidal JC, Stoppani AOM. Isolation and Purification of two Phospholipases A from Bothrops Venoms. Arch Biochem Biophys. 1971; 145: 543-556.

22. Delano WS. The PyMol, Molecular Graphics System. [Internet] Delano Scientific, San Carlos, CA. 2002. [Consultado mayo 2019]. Disponible en: http://www.pymol.org 